\title{
Controlled Light-Driven Levitation of Macroscopic Plates
}

Mohsen Azadi, George A. Popov, Zhipeng Lu, Andy G. Eskenazi, Avery J.W. Bang, Matthew F. Campbell, Howard Hu, and Igor Bargatin*

*Corresponding author: bargatin@seas.upenn.edu 

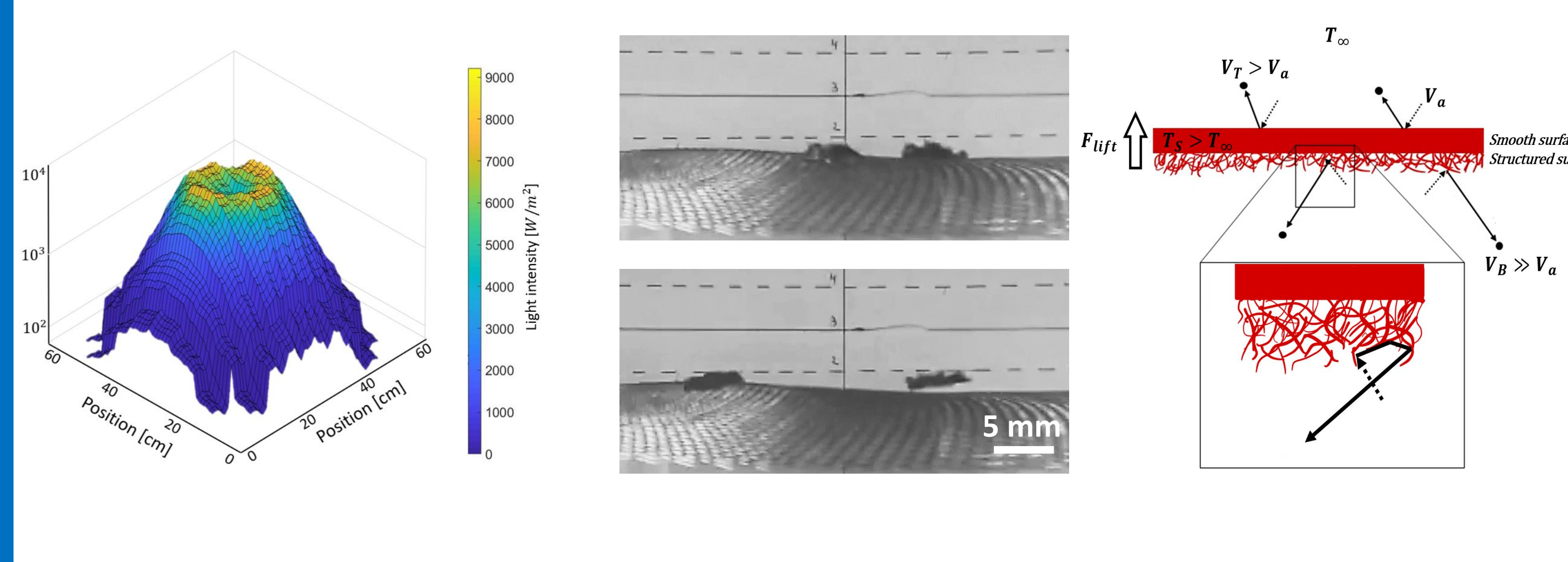
face 


\section{Controlled Light-Driven Levitation of Macroscopic Plates}

We report light-driven levitation of 0.5 -um thick mylar samples that have been modified by depositing a 300-nm-thick layer of carbon nanotubes (CNTs) on a single side. The CNT layer serves three key purposes: 1) It acts as a lightweight light absorber, absorbing $\sim 90 \%$ of the incident light and elevating the temperature of the sample. 2) It increases the structural rigidity of the mylar film, allowing cm-scale discs with submicron thicknesses to hold their shape. 3) It creates a structured porous surface that traps impinging gas molecules, which results in an accommodation coefficient difference between the top and bottom surfaces for gas-surface interactions. Air molecules that rebound from the CNT-coated side have on average higher velocities than those departing from the opposing uncoated mylar surface. We show that the net force thus created can be used to levitate the mylar films. Moreover, we will demonstrate our ability to manipulate a light field in order to control the flight of levitating samples for extended periods of time.

Keywords: Photophoresis, light-driven motion, near-space flight

M. Azadi, G. A. Popov, Z. Lu, A. G. Eskenazi, A. J. W. Bang, M. F. Campbell, H. Hu and I. Bargatin, Controlled levitation of nanostructured thin films for sun-powered near-space flight Sci Adv 7 (7), eabe1127. DOI: 10.1126/sciadv.abe1127 
Micromachines Conference April 2021

Controlled flight of a $1 \mathrm{~cm}$ disc over a large light trap $60 \mathrm{~Pa}$ environment 
 \\ Engineering

\section{Inaccessible regions of the Earth's atmosphere}
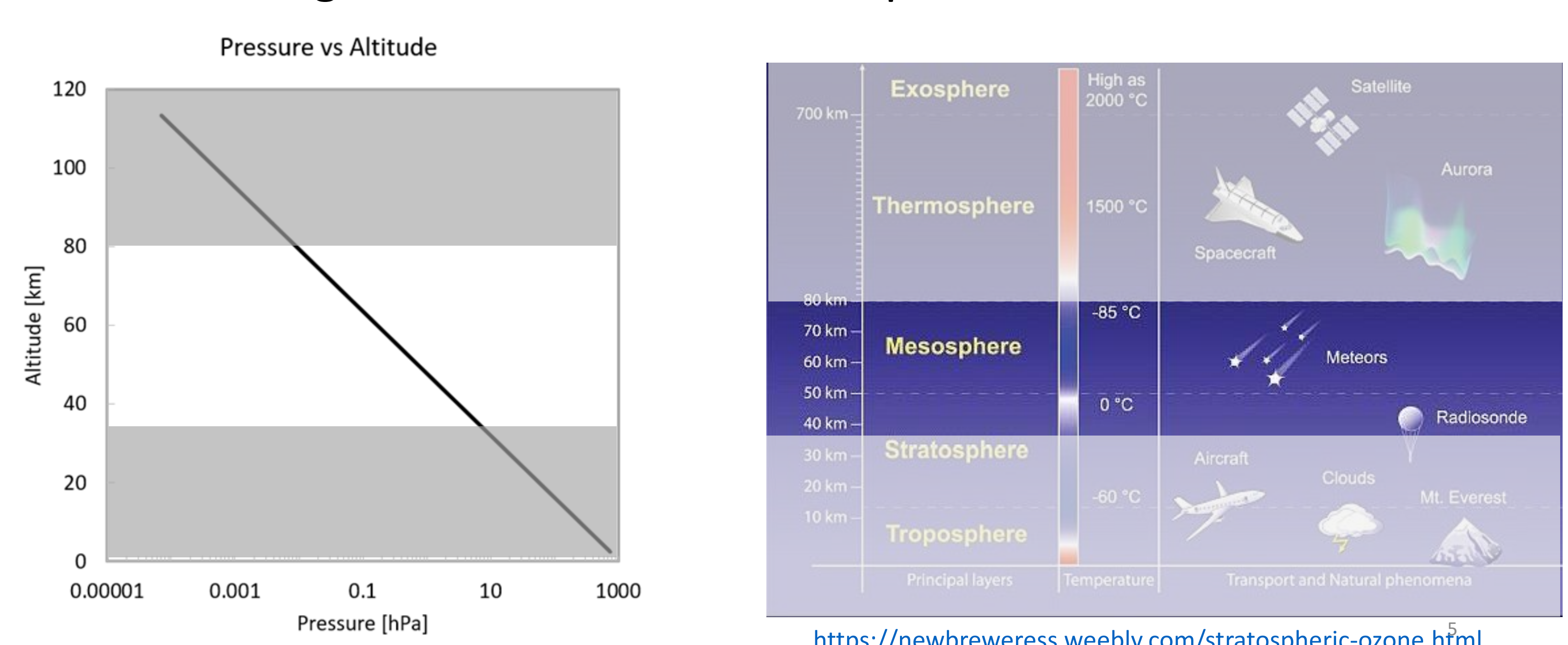

https://newbreweress.weebly.com/stratospheric-ozone.html 


\section{Inaccessible regions of the Earth's atmosphere}

- No flight mechanisms available

- Important for weather, sensing, surveillance, etc.

Low ambient pressure for aircraft and balloons, high pressures for satellites causing high drag force.

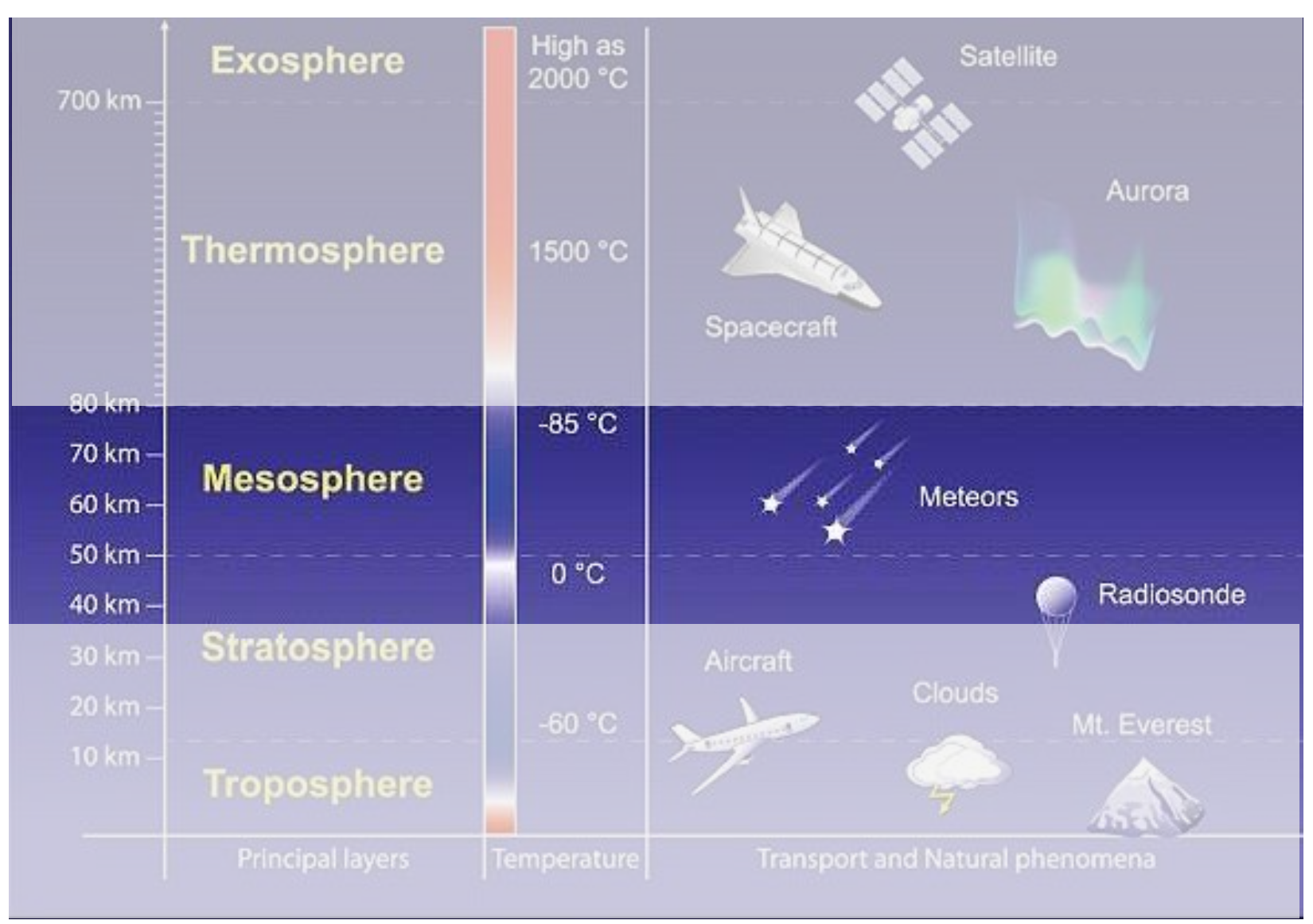


Photophoresis on microscopic particles:

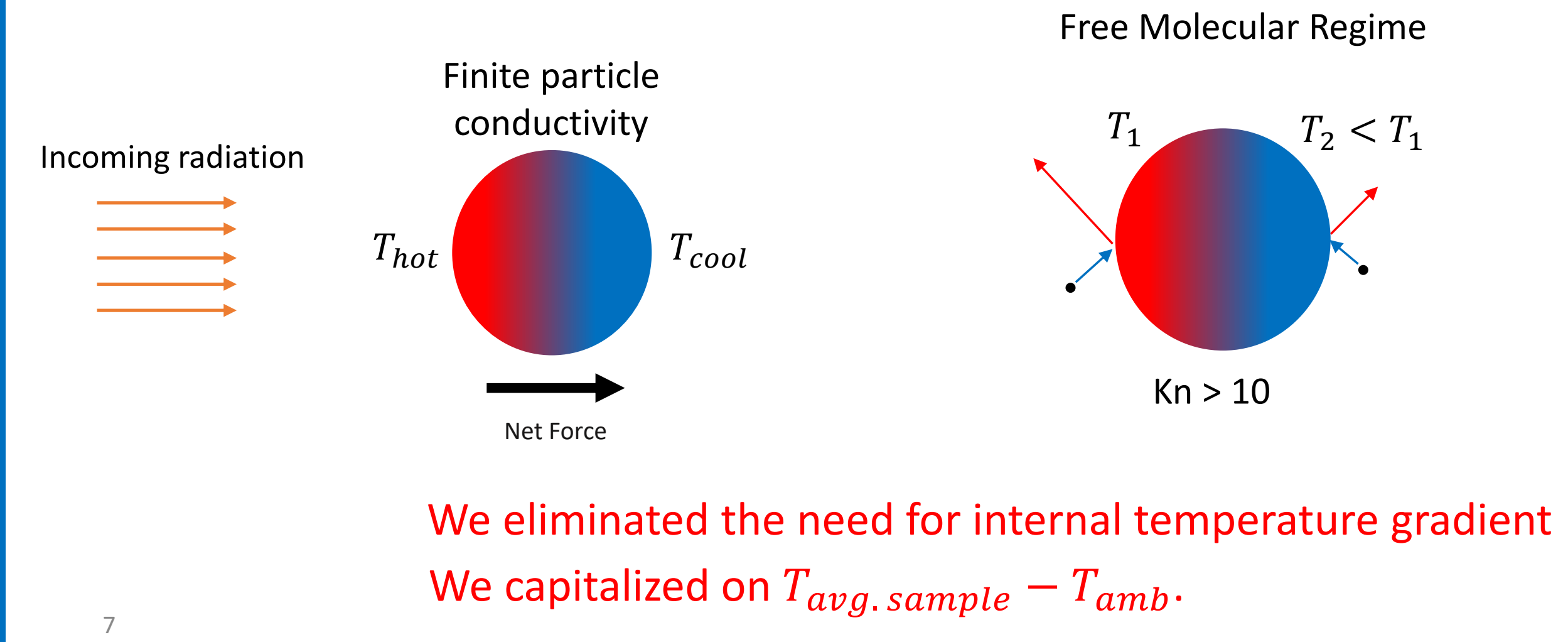

Slip Flow Regime

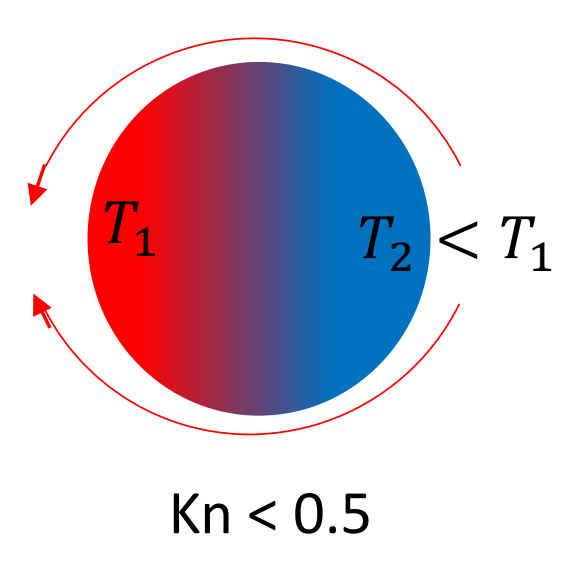

We eliminated the need for internal temperature gradient

We capitalized on $T_{\text {avg. sample }}-T_{a m b}$. 
Assume we have a uniformly heated sample: thin disk

Consider a very thin plate in a low-pressure gas where intermolecular collisions don't happen.

$$
\alpha=\frac{T-T_{\infty}}{T_{S}-T_{\infty}}
$$

Thermal accommodation coeff.: $\alpha$ Indicator of thermal interaction between gas molecules and surface
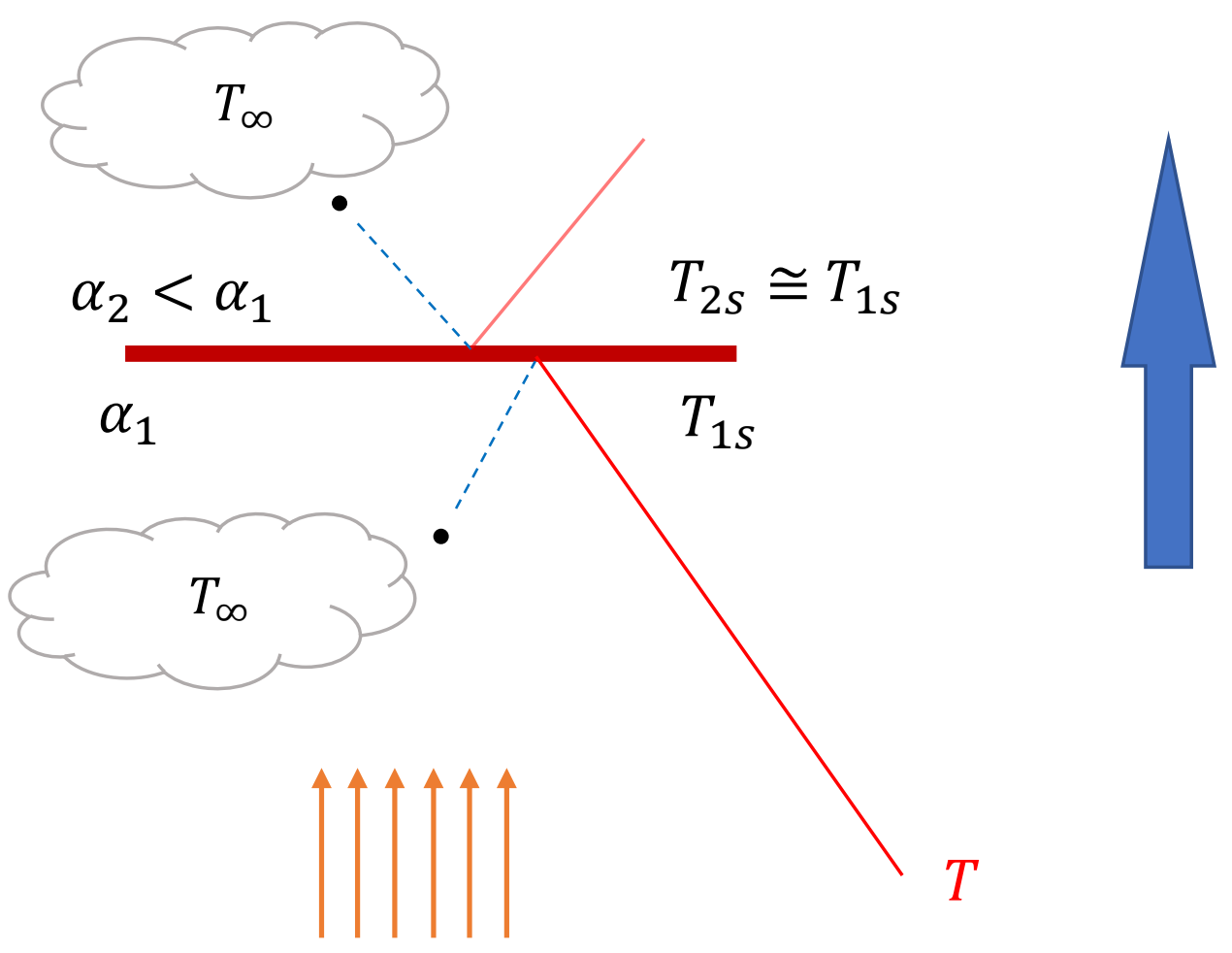

Net Force on the Plate

$\Delta \alpha$-Force 

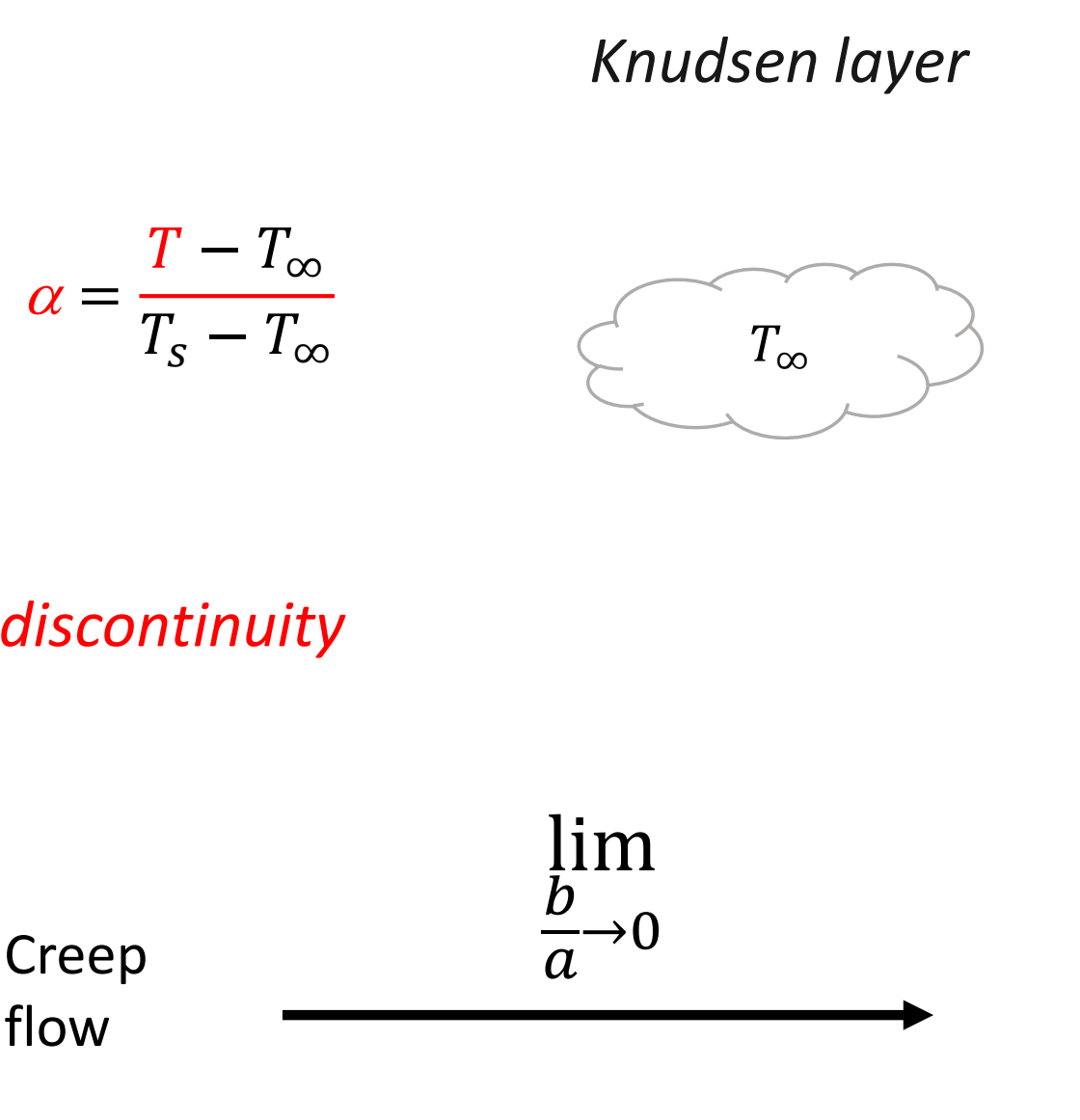

Slip flow regime; physics:
\[ \alpha=\frac{T-T_{\infty}}{T_{S}-T_{\infty}} \]

Slip flow regime; Formulation: Avoiding discontinuity

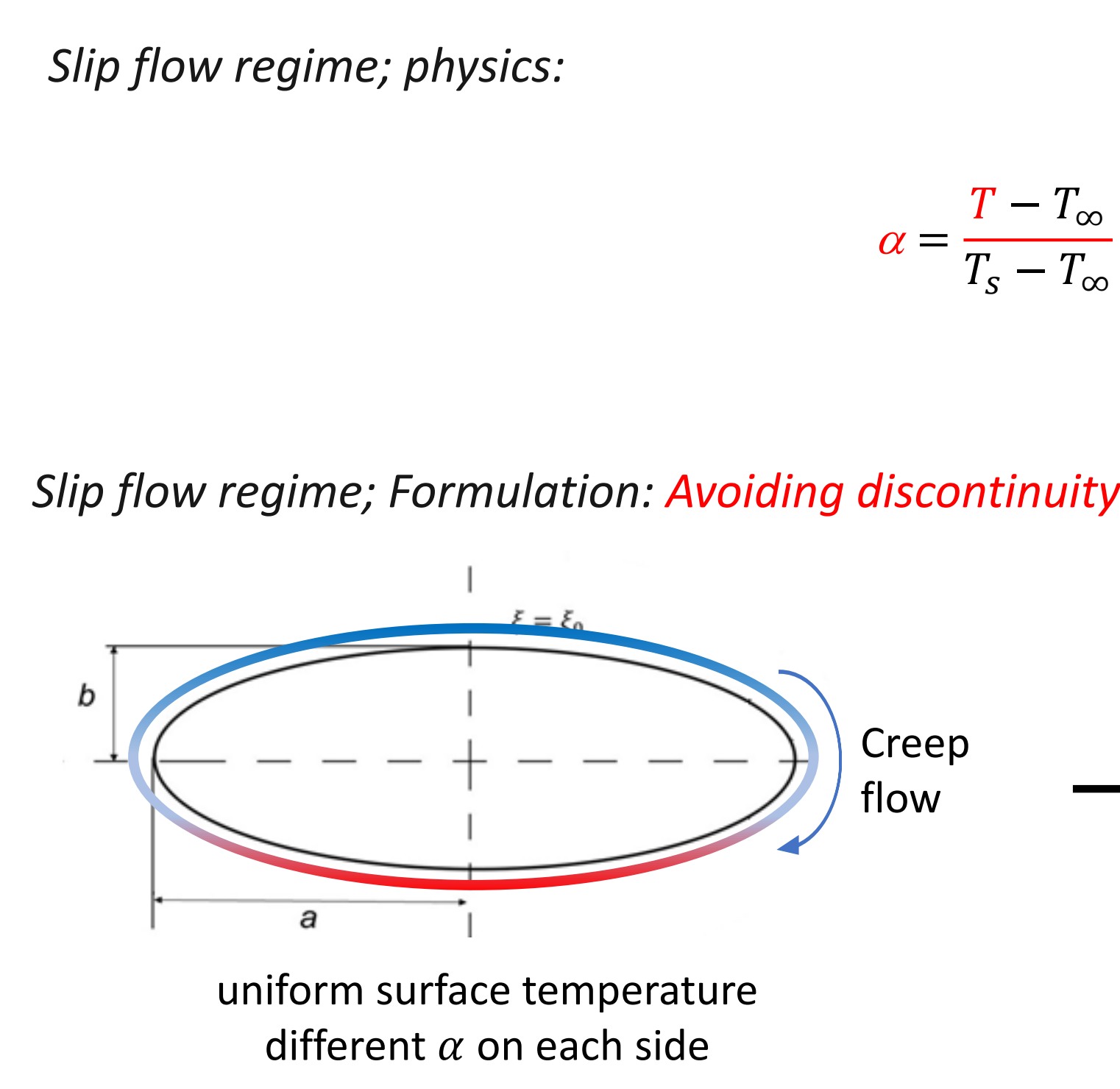

uniform surface temperature different $\alpha$ on each side

$$
\alpha=\frac{T-T_{\infty}}{T_{s}-T_{\infty}}
$$

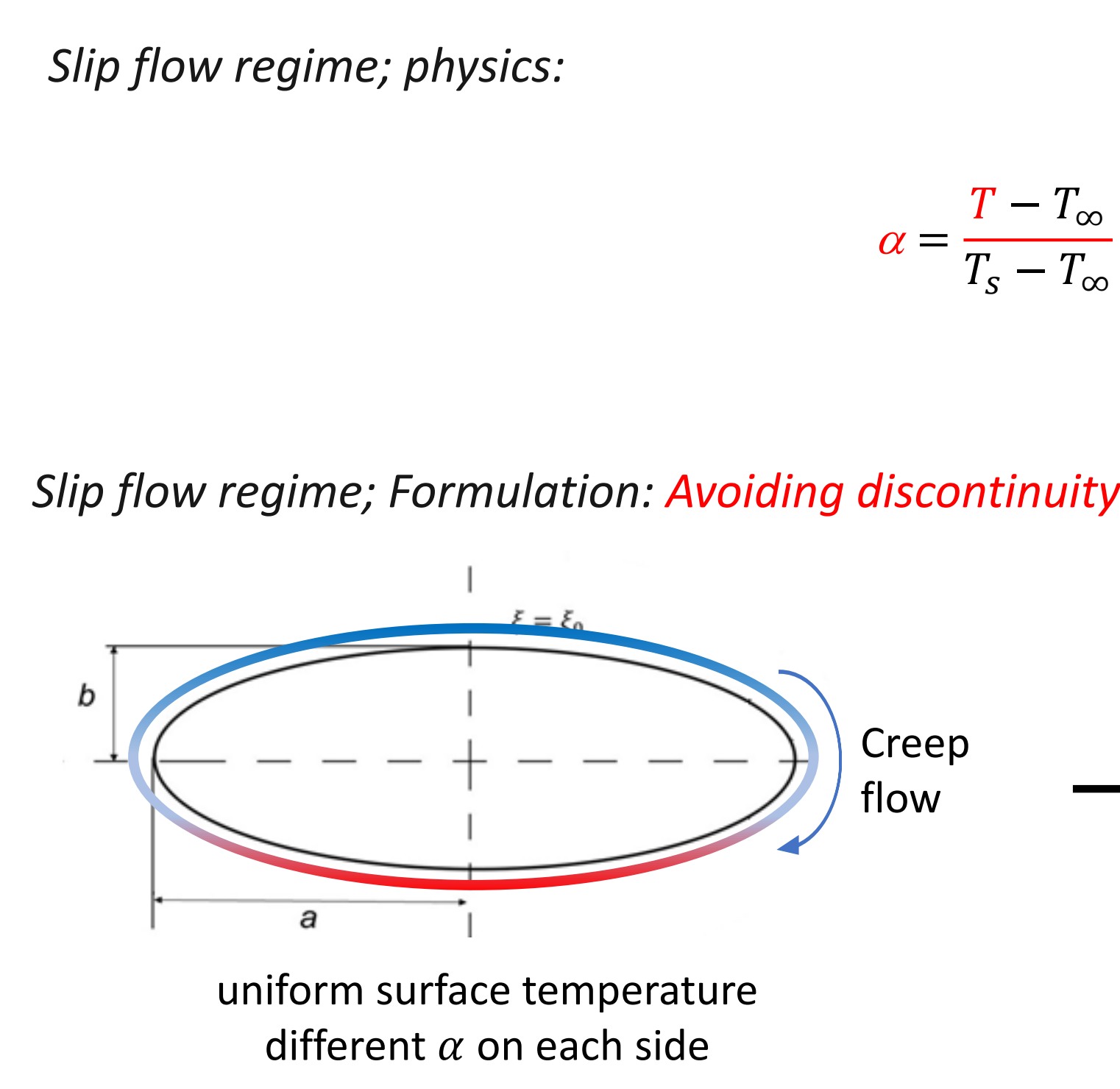

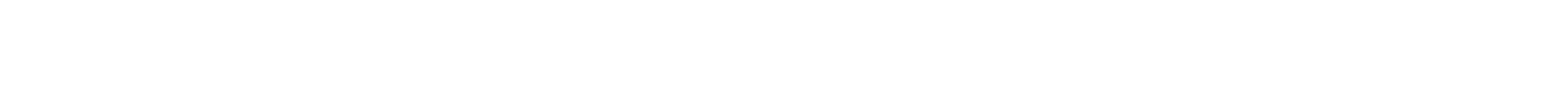
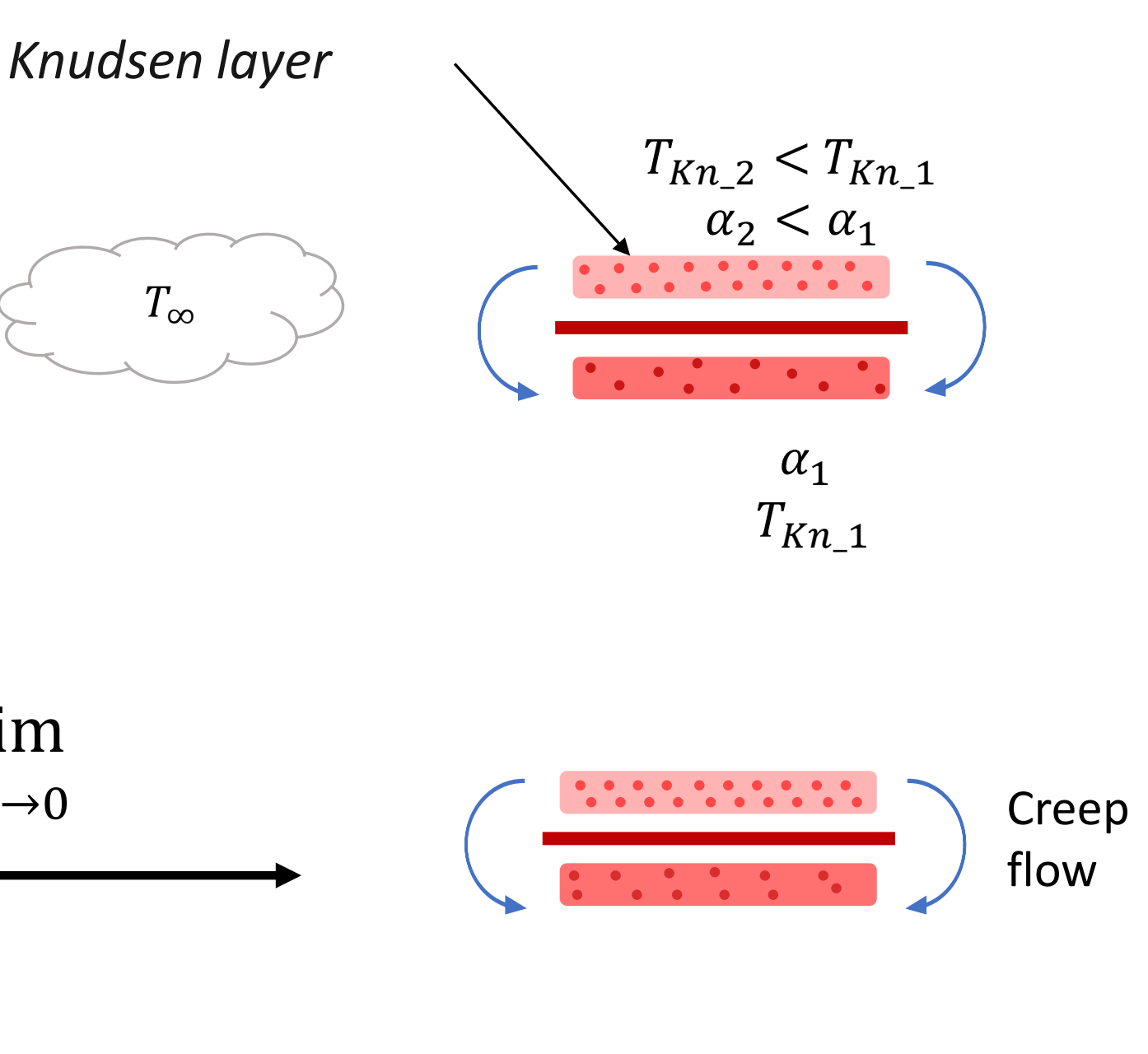

$\alpha_{1}$

Knudsen layer

$$
T_{K n_{-} 1}
$$

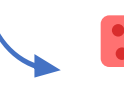

西

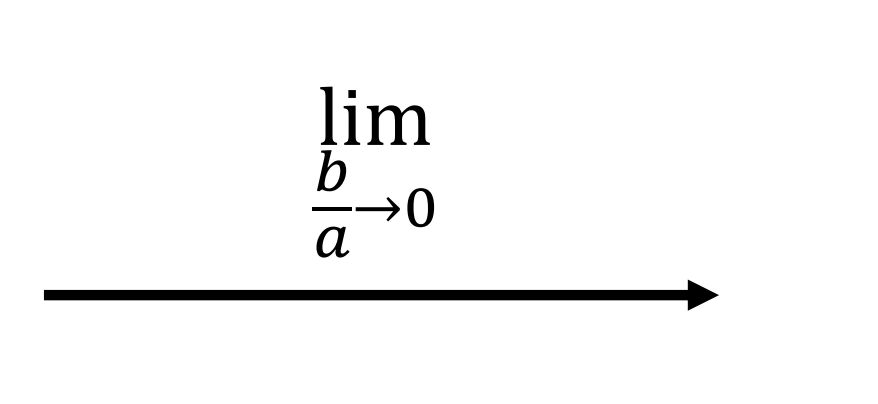

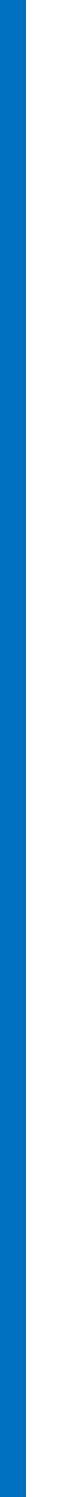

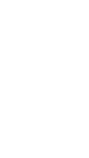

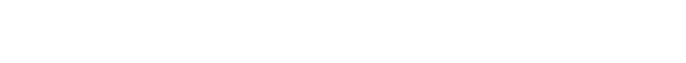


$T_{\infty}$

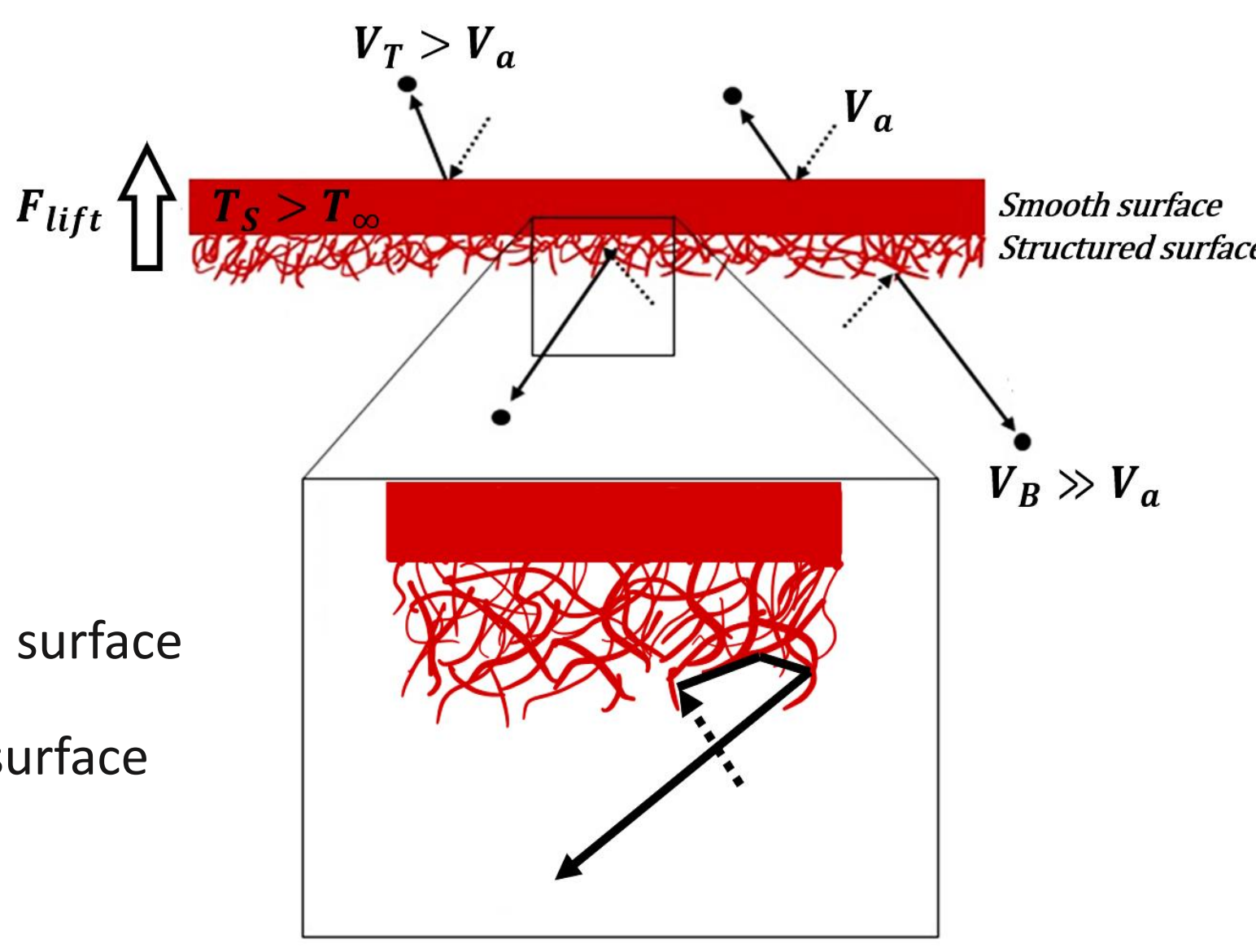

$V_{B} \gg V_{a}$

Cheap, fast, easy to make samples:

What we MUST have: $\quad \Delta \alpha$ between the two sides

$$
\alpha=\frac{T-T_{\infty}}{T_{S}-T_{\infty}} \sim \begin{gathered}
\text { how much energy the molecule } \\
\text { absorbs from the surface }
\end{gathered}
$$

Easy ways to change $\alpha$ :

- Change the interaction time between gas molecule and surface

- Increase number of collisions each molecule has with surface before returning to ambient

\section{Creating a trap}




\section{Sample Fabrication}

Cheap, fast, easy to make samples: Creating a trap

Water based CNT solution

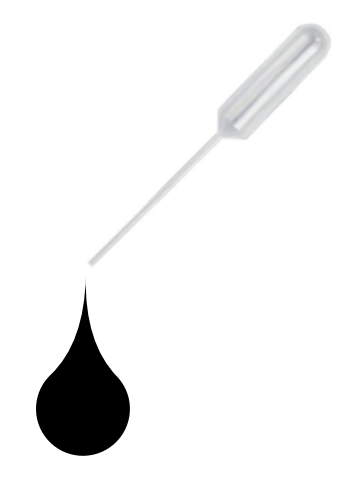

4" wafer after evaporation of the water:
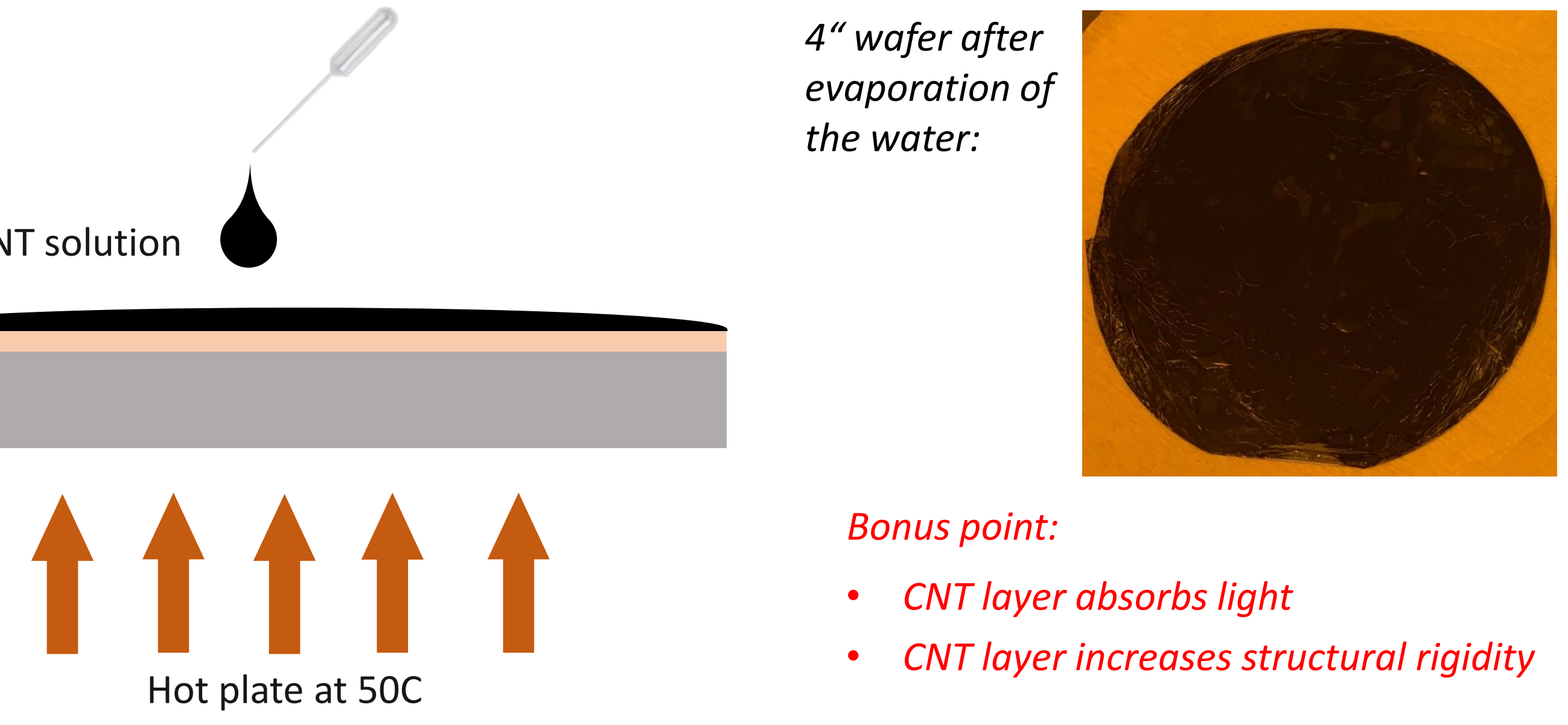

Bonus point:

- CNT layer absorbs light

- CNT layer increases structural rigidity 


\section{Engineering

\section{Penn \\ Sample Fabrication}

Cheap, fast, easy to make samples: C Creating a trap

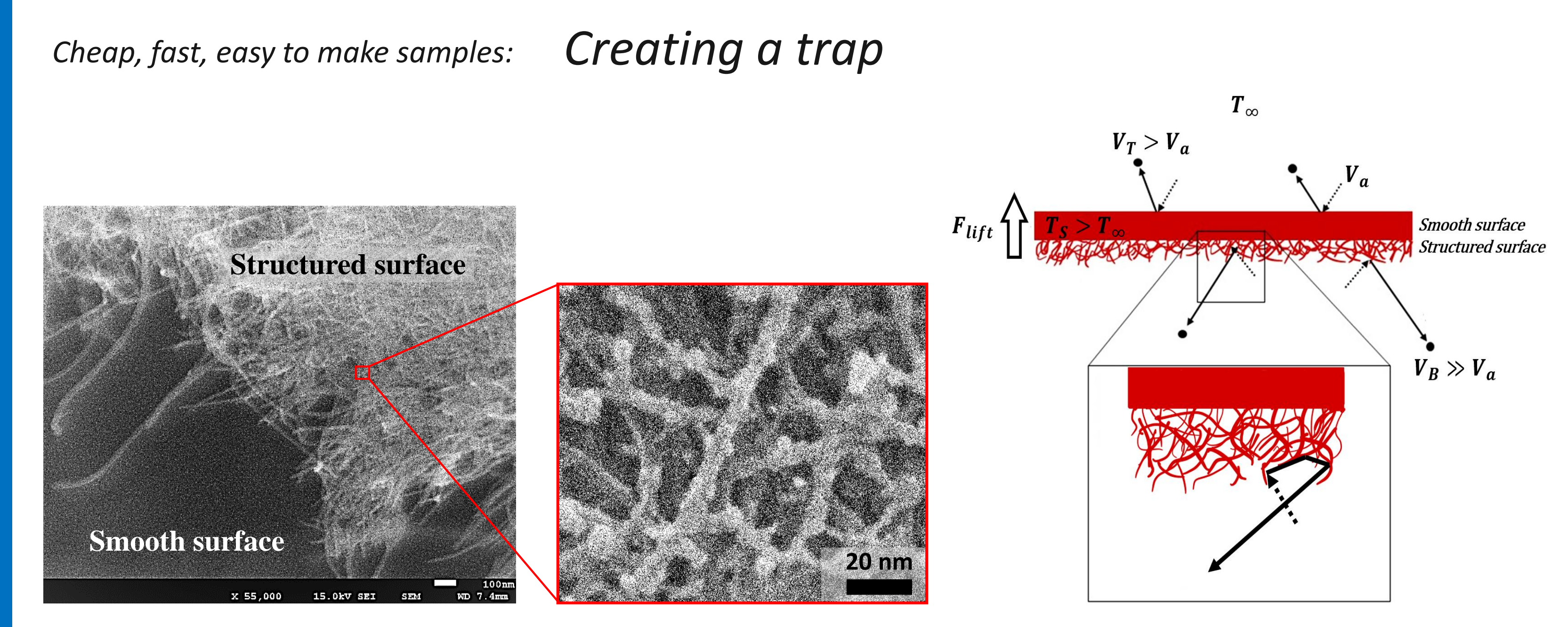

GROUP

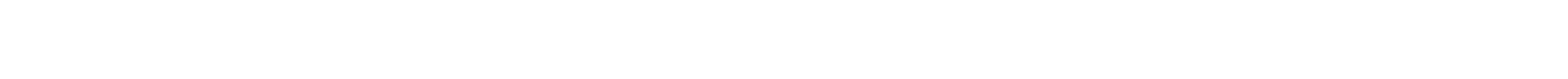


Creating a light trap and the experimental setup:

- 8 LED setup for a light ring - up to 10 suns in intensity of the center of the ring.

- Transparent metallic mesh (74\%) as launch pad

- Clear acrylic chamber for video capturing
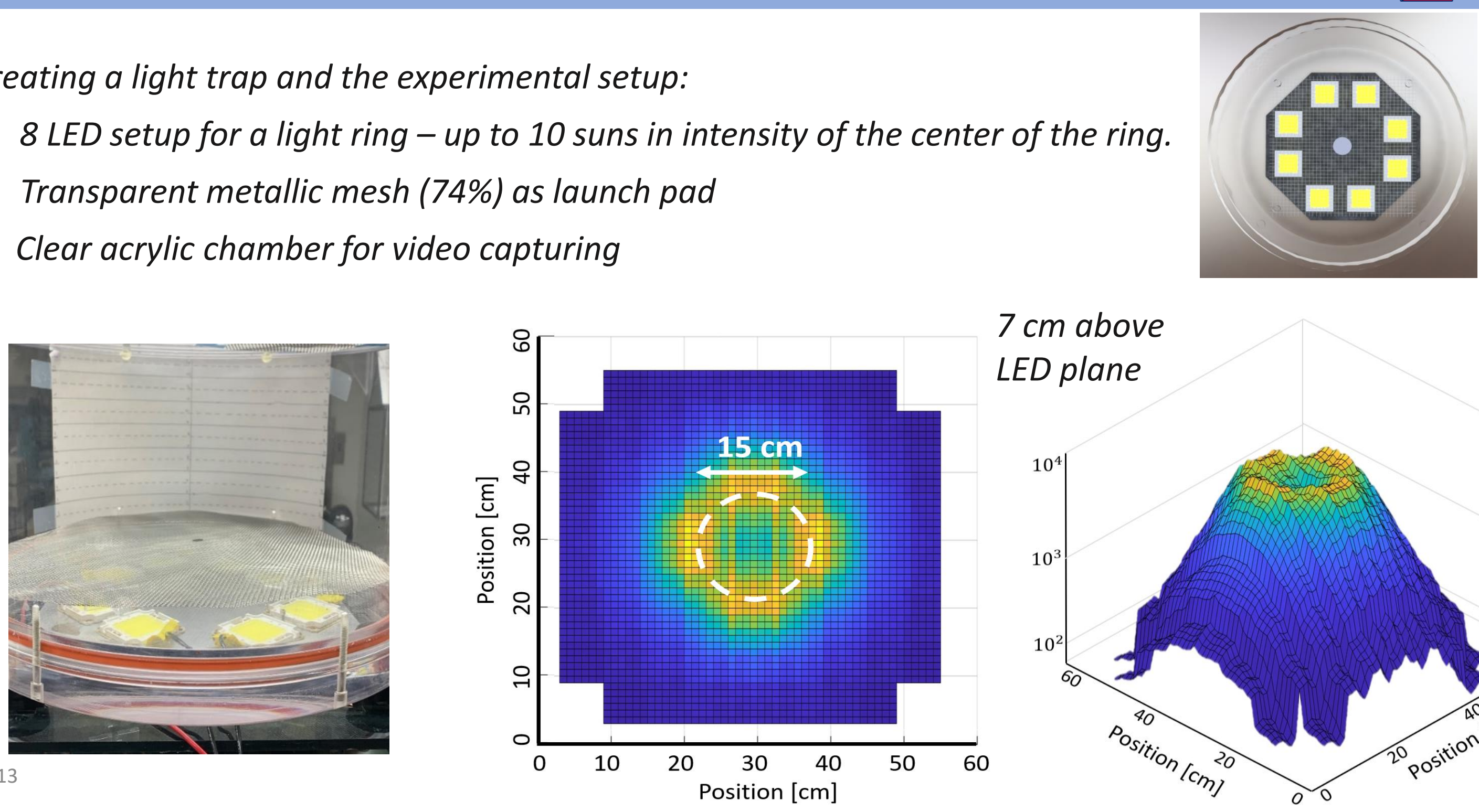

\section{$7 \mathrm{~cm}$ above} LED plane

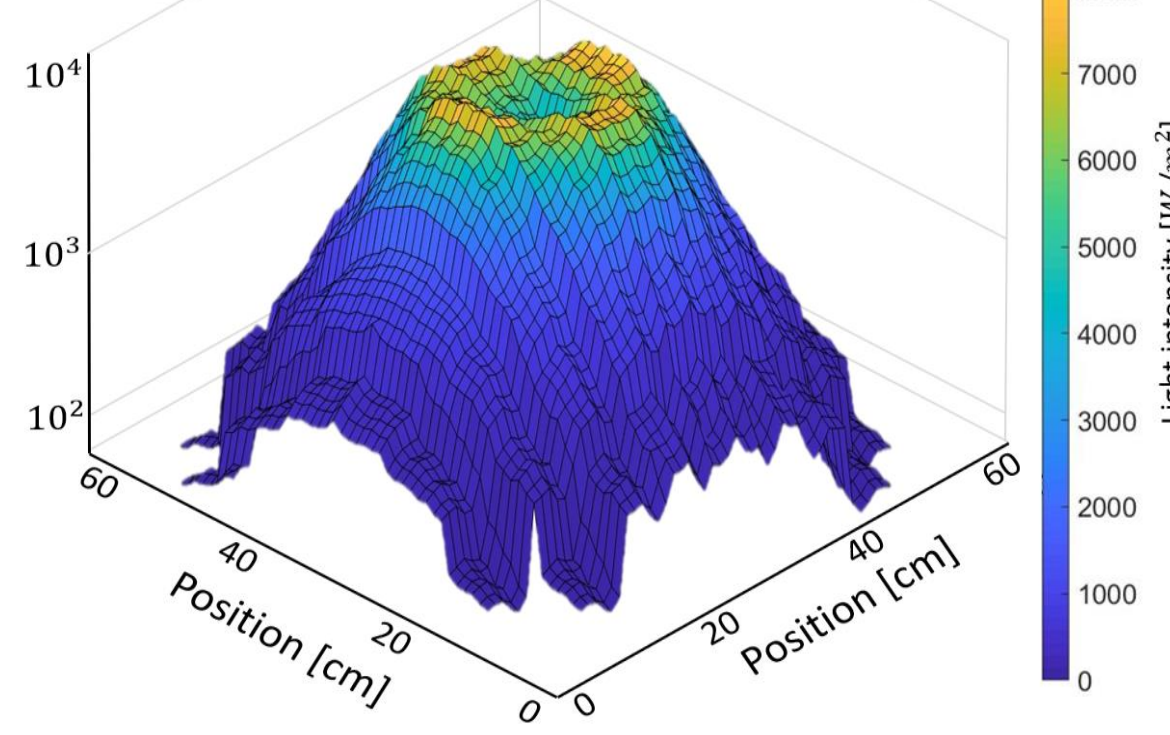


We also tested scenarios that the theory says they shouldn't work:
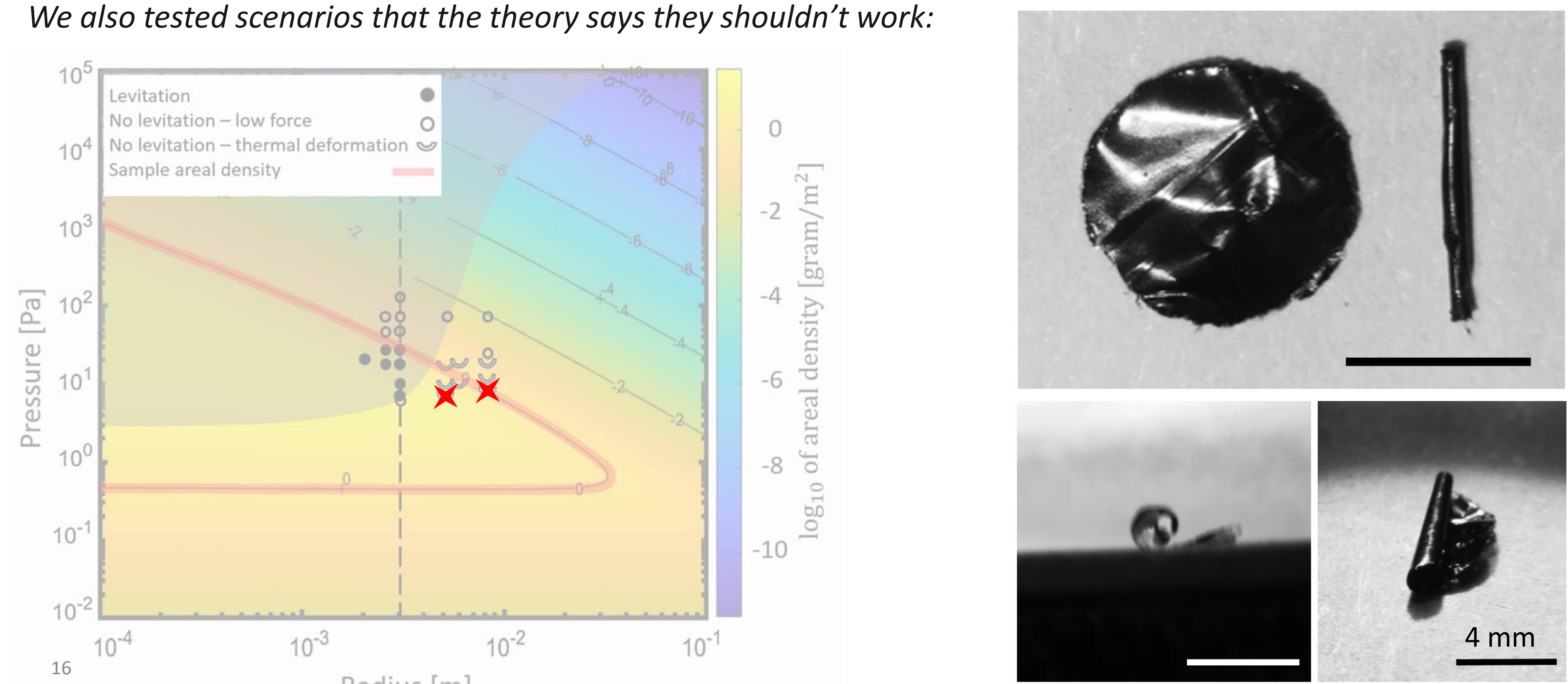 \\ Pent Penn Experimental results}




\section{Earth's upper atmosphere:}

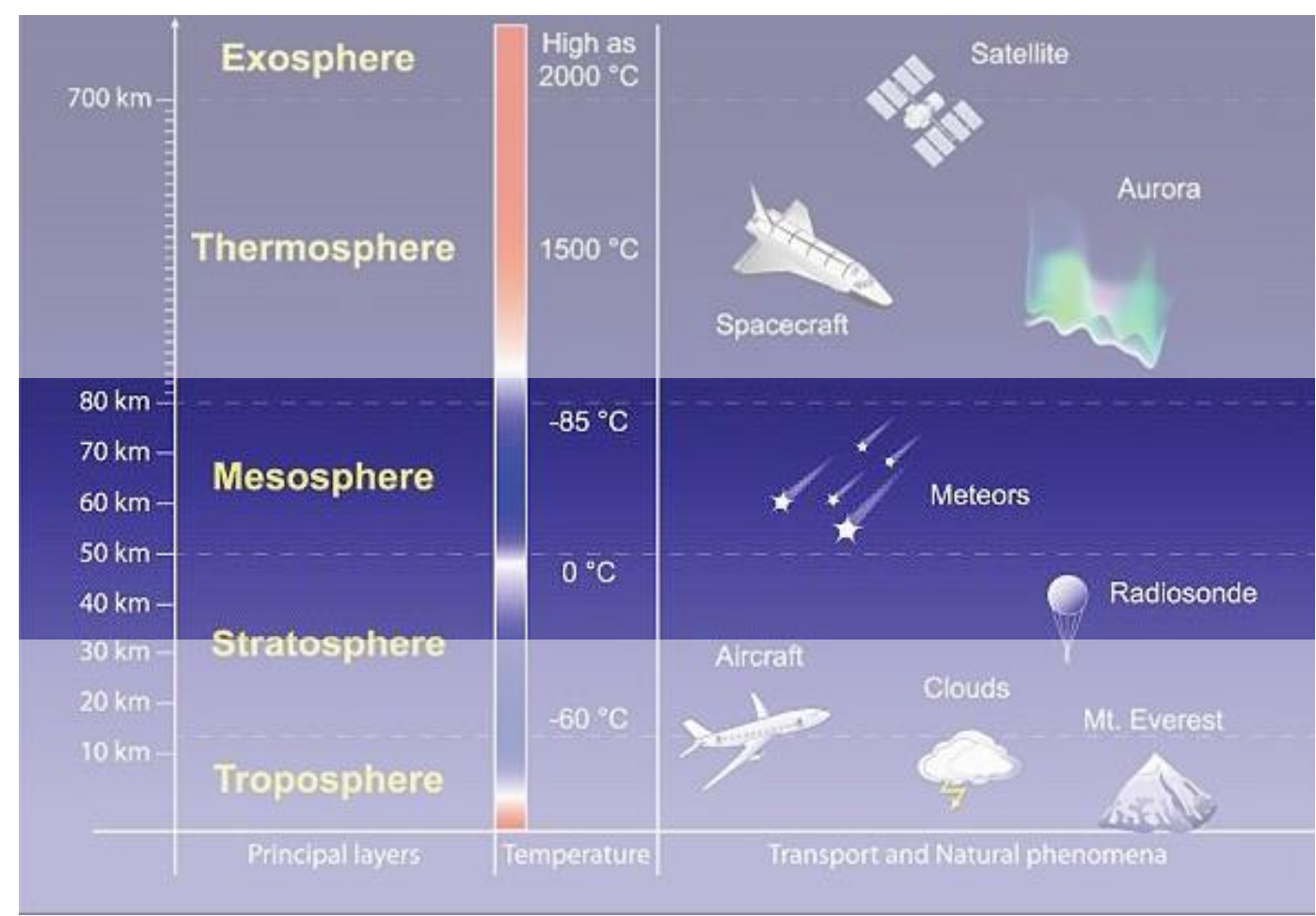

https://newbreweress.weebly.com/stratospheric-ozone.html
Optimized values of $\Delta \alpha=0.5$ and emissivity= 0.5 under natural sunlight
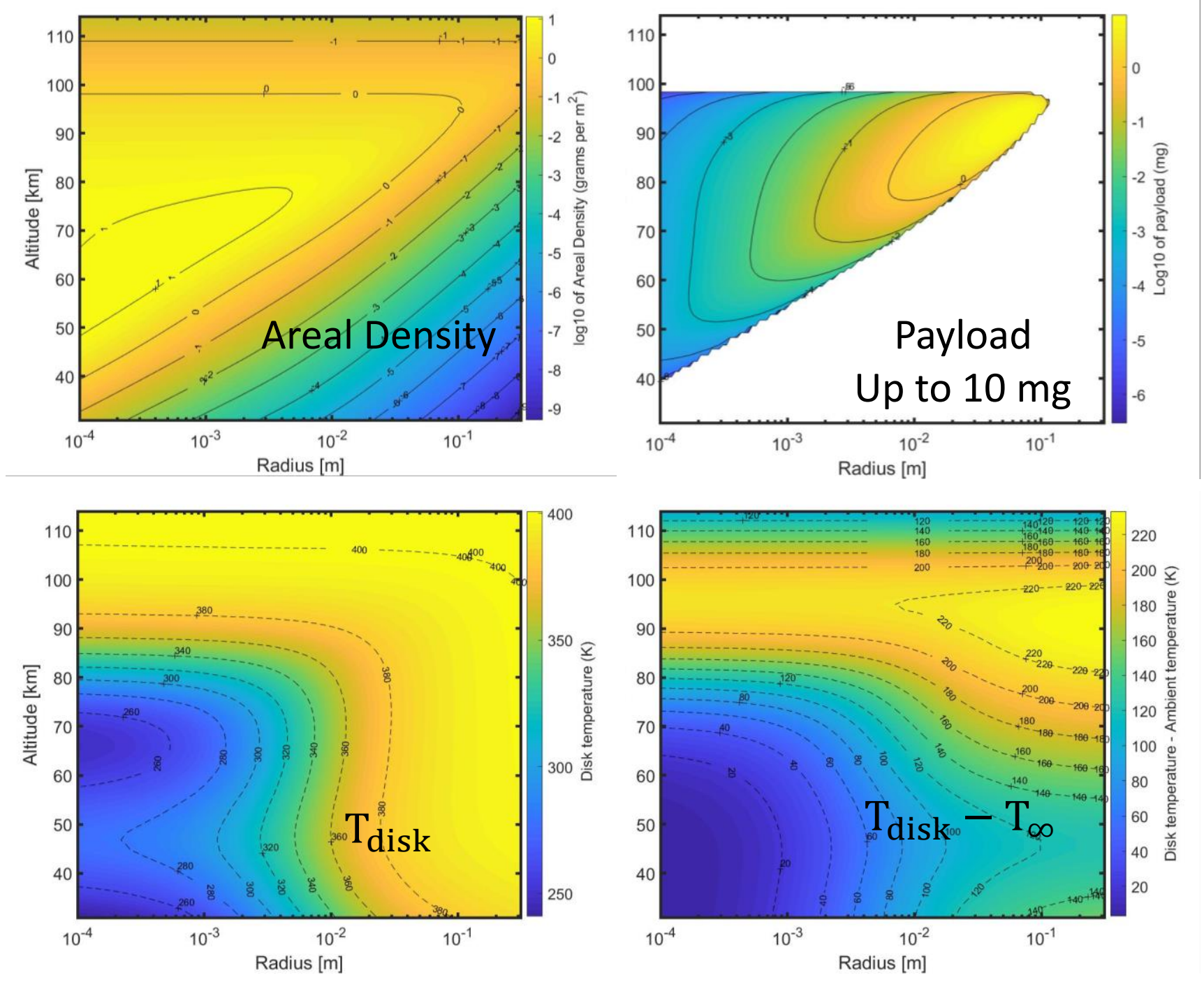
We thank the staff of the Singh Center for Nanotechnology, Nanoscale Characterization Facility, and Scanning and Local Probe facility at the University of Pennsylvania, especially Meredith Metzler, and Eric Johnston.

The Singh Center for Nanotechnology, Nanoscale Characterization Facility, and Scanning and Local Probe facility at the University of Pennsylvania is partly funded by the NSF National Nanotechnology Coordinated Infrastructure Program, under grant NNCl-1542153. This work is partly supported by the NSF under CBET-1845933 and the School of Engineering and Applied Science at the University of Pennsylvania 
